\title{
Handwheel valve operation: assessment of four opening methods in terms of muscle loading, perceived comfort, and efficiency
}

\author{
Fereydoun Aghazadeh, Saif Al Qaisi, Francis Hutchinson, and Laura Ikuma \\ Department of Construction Management and Industrial Engineering, Louisiana State University, 3128 Patrick F. \\ Taylor Hall, Baton Rouge, LA, USA 70803
}

\begin{abstract}
Injuries due to manual valve handwheel operations are a major concern in the chemical process industry. Manual valves are used to start, stop, and control flow in process lines. The torque required to operate handwheels after the initial "breaking" can vary due to the size, design, and condition of the valve. This study tested four methods of turning the handwheel on a medium-sized gate valve at two different torque requirements $(25 \mathrm{Nm}$ and $50 \mathrm{Nm}$ ). The methods were compared to each other, in terms of efficiency, subjective ratings of perceived exertion, and electromyography activity of the upper extremity and trunk muscles. The results indicate that different methods and torque requirements are compensated for by the use of different muscles. Also, this use of different muscles, along with the increased mechanical advantage of the wrench, can affect the operator's actual and perceived comfort and efficiency while operating a valve handwheel.
\end{abstract}

Keywords: valve operation, handwheels, EMG, torque, MSDs

\section{Introduction}

Industrial valve handwheels are in common use in many industries. Petroleum, power generation, chemical, and waste process plants utilize handwheel actuated valves to block, allow, or regulate the flow of materials within a system [10].

Although much research has been done to determine the torque capabilities and optimal handwheel positioning [3], the literature reveals little has been done to investigate the dynamic forces and strains involved in handwheel actuation [1]. Opening and closing valve handwheels has been determined to be a significant cause of serious shoulder injury at refineries [11]. Parks and Schultz [7] report that 56\% of low back injuries and $75 \%$ of head, neck, and face injuries reported by process operators at a large corporation over a three-year period were attributed to industrial valve handwheel actuation. Additionally, a musculoskeletal discomfort questionnaire administrated by Amell [1] indicates that $88 \%$ of process operators at a large petroleum refinery believed that the musculoskeletal discomfort they experienced was attributable to their job. The operators also believed that industrial valve handwheel actuation was the most physically demanding task they were required to perform [1].

In addition to potential musculoskeletal injuries, there are possible cardiorespiratory risks in valve operations as well. Meyer et al. [6] measured heart rate and oxygen uptake during continuous handwheel actuation and found results of cardio respiratory strains. This testing was done simulating opening/closing a valve one time, at a relatively low torque resistance. The results led the authors to recommend that controls be instituted to reduce the possibility of excessive cardiac demand under actual working conditions.

Most of the research on hand wheel turning has focused on static torque studies. These usually measure a maximum isometric force exerted by the operator and are useful in studying the problems with the breaking force required. The breaking force is the force required to start the initial movement of a handwheel from a locked position to an unlocked position. Parks and Schulze [7] studied 336 valves of 
various handwheel diameters and heights at a large petroleum refinery and found that the breaking force* to open a handwheel ranged from $100 \mathrm{Nm}$ to as high as $225 \mathrm{Nm}$. Other studies looked at the torque production capabilities of operators and found that the maximum torque exerted by the operators was 62 $\mathrm{Nm}[1,9,10]$, which is significantly less than the maximum and even the minimum torque demands mentioned earlier. Similarly, Jackson et al. [4] found that $93 \%$ of 217 valves in a chemical plant required a torque over $400 \mathrm{Nm}$. Even the Guidelines for Manual Operation of Valves approved by the Manufacturers Standardization Society of the Valve and Fittings Industry (MSSVFI) exceed the capabilities of most workers. The guidelines assume that the peak dynamic torque for a handwheel with a diameter of $46 \mathrm{~cm}$ (18 in) is greater than $230 \mathrm{Nm}$ (MSSVFI, 1996), which is far greater than the maximum torque exerted by the operators mentioned earlier $(62 \mathrm{Nm})$.

After the initial breaking force, the operator is required to put continuous effort in actuating the handwheel. This continuous muscular effort, for as much as five to fifteen minutes, is believed to place greater demands physically and physiologically on the operator than the initial breaking torque requirement [4]. In some cases, such as in starting up or shutting down a unit, the process plant operator may be called upon to open or close as many as 50 to 100 valves, during the same working shift. Although continuous handwheel actuation is more demanding, there is much less research in this area than in initial handwheel actuation (breaking) [4,6].

When a valve's breaking force is very high or a large dynamic turning force is required, the process operator often has a wrench available to aid in turning the wheel. These wrenches are fairly standard in design and operation and consist of a handle with a jaw on the end that can be hooked over the valve wheel. The length of the wrench provides the operator with a mechanical advantage in turning the wheel. Since valve wheel wrenches are widely used in valve operations, research of the dynamic forces and strains in handwheel actuation should include evaluations of these tools.

This study was designed to evaluate and compare four different techniques of continuous handwheel actuation, in terms of efficiency, comfort, and amount of muscle loading on the upper extremity and trunk muscles. Two of the techniques are conventional methods in handwheel actuation, which are the use of bare hands only and the use of a regular

\footnotetext{
* Corresponding author. aghazadeh@su.edu
}

wrench. The third technique involves the use of a modified wrench in turning the wheel. The modified wrench was used as a prototype to determine if this type wrench would be effective in reducing the stresses encountered when operating a valve handwheel.

\section{Methodology}

\subsection{Participants}

Eight participants in good health between the ages of 18 and 45 were tested. Prior to the data collection, the experimental procedures and the demands of the testing were explained to the participants and their signatures were obtained on informed consent forms approved by the local institutional review board (IRB).

\subsection{Equipment}

A standard, 6" inside pipe diameter manual gate valve was obtained and mounted in a stand with a 13 " diameter hand wheel horizontally-oriented at a height of about $100 \mathrm{~cm}$ from the grade (Figure 1). Although there is no set height for these valves, several studies show that this valve wheel height falls within the ergonomic recommendations $[7,10]$.

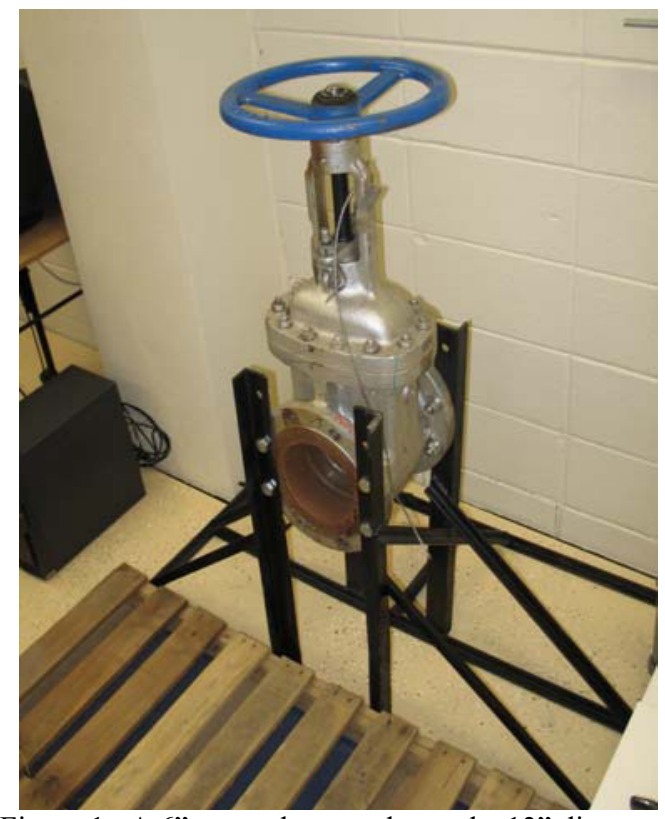

Figure 1. A 6" manual gate valve and a 13" diameter handwheel 
Two types of wrenches were used in this study: (1) a conventional, forged aluminum "crow's foot" valve wheel wrench and a (2) modified wrench. The modification added to the conventional wrench a "spinner" type handle. This was done by fabricating a hinge in the handle to allow the wrench to be hooked on the valve wheel and turned $360^{\circ}$ without having to remove the wrench from the valve wheel. Figure 2 shows a conventional valve wheel wrench and the modified version.

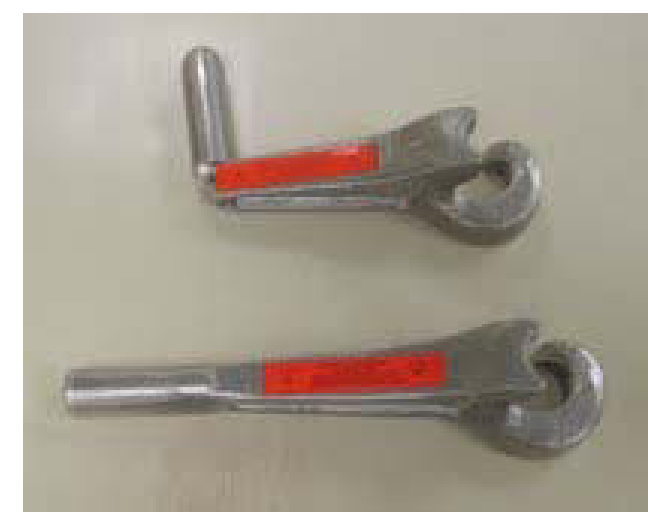

Figure 2. Conventional valve wheel wrench on bottom and modified wrench on top

\subsection{Experimental task}

The participants were instructed to continuously actuate a handwheel valve as fast as possible from fully closed to fully open (counterclockwise), using four different techniques. This protocol was set to simulate a real world situation in which an operator must open or close a number of valves quickly during a shutdown or startup of a production unit. This situation is not uncommon in some production units and is necessary to curtail process or equipment problems that may affect the quality of the product or could potentially lead to a hazardous incident. The testing was done under two different torque settings, $25 \mathrm{Nm}$ and $50 \mathrm{Nm}$. The valve wheel was set at a fairly low torque (approximately $25 \mathrm{Nm}$ ) to simulate a new or well-maintained valve with little or no corrosion, contamination, or other factors to increase the necessary turning force. Also, a higher torque was used to simulate a handwheel-valve system that is still in a fairly good condition but had the valve packing tightened to prevent leakage around the stem (approximately $50 \mathrm{Nm}$ ). This torque requirement is not unusual in the field. Much higher torques are often found in actual operations $[4,7,11]$. The $50 \mathrm{Nm}$ set- ting was based on the Manufacturers Standardization Society guideline MSS SP-91-2009. Torques higher than $50 \mathrm{Nm}$ were not tested to minimize the risk of injuries.

The participants were tested at each of the torque settings using two hands only, a standard valve wrench, and the modified wrench.The standard wrench was used in two different ways simulating the following conditions: (1) an area with obstructions that get in the way of actuation, limiting the amount of turning; and (2) an area without obstructions. The first condition was simulated by having the participants turn the valve wheel about $2 / 3$ of a revolution, unhooking the wrench from the valve wheel, and repositioning it as if there was an obstruction limiting the amount of turning. The second condition was simulated by having the participants continuously turn the handwheel all the way around with the wrench. Obstructions are commonplace as pipes are usually routed together, and it is often difficult to have unobstructed access to a valve. Hence, there were a total of 8 trials, and the order of the trials was randomized. Participants were allowed to rest between trials until their heart rate returned to the resting rate. In all the trials, they were not required to "crack" the valve open. Preliminary tests indicated that "cracking" forces can vary from trial to trial on the same valve. Also, "cracking" forces of high torques may affect the performance of some participants. So to eliminate cracking force exertions, the handwheel was opened approximately $1 / 8^{\text {th }}$ of a turn from the fully closed position. Participants were asked to perform each trial as fast as possible to simulate "real world" conditions as when a hurried or emergency condition is called for and numerous valves must be opened or closed quickly.

\subsection{Data collection}

All trials were timed starting from the valve in the fully closed position to the fully open position. The time measurements were taken to compare the efficiency of the different techniques.

Also, after the completion of each task, participants were asked to rate their perceived exertion of the task using Borg's category-ratio based CR10 scale. Perceived exertion is defined as the subjective intensity of effort, strain, discomfort, or fatigue experienced during physical exercise [8]. All the participants were given a brief introduction to the use of ratings of perceived exertion (RPE) and an explanation of the categories described on the CR10 scale. 
In addition to the subjective data, objective data was also collected on the muscle loading of the upper extremity and trunk muscles. Muscle activity measurements were recorded using an EMG Bagnoli 8channel system by Delsys of Boston, MA. The electrodes used were surface mounted electrodes that were affixed to the skin by interface tape which had adhesive on both sides. Before attaching the electrodes, the surface of the skin at the areas of interest was cleaned to reduce noise in the signal and improve accuracy.

Based on preliminary observation, the electrode placement sites were chosen to be at the right and left biceps, right and left deltoids, right and left trapezii, and right latissimus dorsi muscles.

The signal was normalized with respect to the maximum voluntary contraction (MVC) of each muscle. The data from the trials was then filtered to obtain the maximum average value for the highest muscle activity during the trials. Then this resultant was averaged to obtain the mean absolute value (MAV) for each muscle as a percentage of its MVC.

\section{Results}

\subsection{Efficiency of techniques}

The average time to fully open the valve system for each technique and at each torque setting was recorded. Figure 3 shows a bar chart of the average times to complete each trial. This figure illustrates that the regular wrench-restricted took the longest time at both torque settings. It took over 100 seconds to complete the task at both torques. The modified wrench, on the other hand, required the least time to fully open the valve system. At $50 \mathrm{Nm}$, it required close to a minute to complete the task, and at $25 \mathrm{Nm}$, it required less than half a minute to complete. The bare hands technique was the second most efficient technique following the modified wrench. However, the times for the bare hands were not much different than the times of the modified wrench. In summary, the order of the techniques, starting with the most efficient first, would be: modified wrench, bare hands, regular wrench-unrestricted, and regular wrenchrestricted.

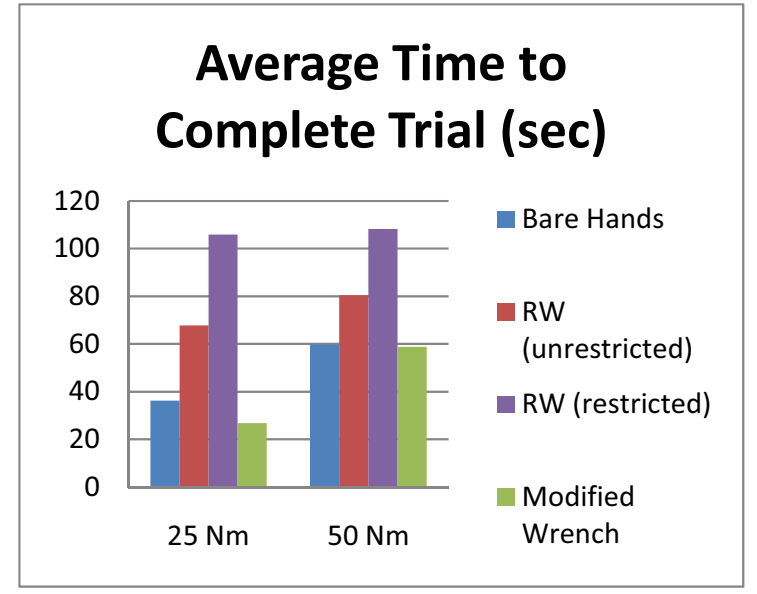

Figure 3. Average time for each technique to fully open valve system.

\subsection{Rating of perceived exertion}

Participants rated the perceived exertion of each technique in opening the valve system. Figure 4 shows a bar graph of the average Borg ratings at both torque settings. At $25 \mathrm{Nm}$, all the methods had approximately the same Borg rating of 3 . However, the modified wrench had the lowest rating right over 2.5. At $50 \mathrm{Nm}$, to the contrary, the modified wrench had approximately the highest Borg rating of 7 along with the bare hands technique. The regular wrench restricted had the lowest rating at $50 \mathrm{Nm}$, which was close to 6 .

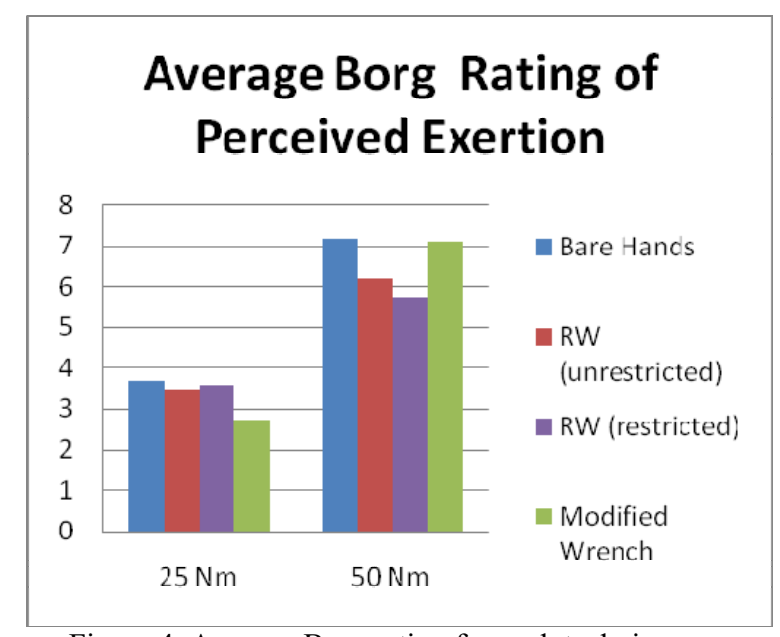

Figure 4. Average Borg rating for each technique. 


\subsection{EMG results}

In all the trials, muscle activity was recorded from the right and left biceps, right and left deltoids, right and left trapezii, and right latissimus dorsi muscles. Figure 5 shows the EMG results of all the muscles of interest for all the trials. The EMG results of each muscle is presented in a graph as a percent of its MVC (\%MVC).

Looking at all the graphs at $25 \mathrm{Nm}$, the regular wrench unrestricted technique shows to have the highest \%MVC for all the muscles, except for the left bicep where it had the second maximum. The bare hands technique had the highest \%MVC signal only once, which was at the left bicep. The regular wrench-restricted and the modified wrench techniques never had a highest \%MVC for any of the muscles at the $25 \mathrm{Nm}$ torque. Further observations from Figure 5 show that the regular wrench-restricted and the modified wrench techniques had the lowest $\% \mathrm{MVC}$ signal at most of the muscles, while the bare hands and the regular wrench-unrestricted techniques never had a lowest \%MVC at any of the muscles. Based on these findings, the order of the techniques, starting with the technique that requires the least overall muscle activity, would be: regular wrench restricted, modified wrench, bare hands, and finally regular wrench unrestricted.

On the other hand, the EMG results at $50 \mathrm{Nm}$ are not as clear on which technique had the lowest/highest overall muscle activity. The modified wrench had near to the highest \%MVC for most of the muscles. It seems to require the most overall muscle activity from all the techniques. The regular wrench unrestricted, seems to have the lowest overall muscle activity as compared to the other techniques.

\section{Conclusions and Discussion}

Subjective and objective data were collected in this study to determine which technique was most comfortable, as well as most efficient, in opening valve systems. At the low torque, the EMG results indicate that the regular wrench restricted required the least overall muscle activity followed by the modified wrench. However, the perceived exertion results at $25 \mathrm{Nm}$ show that the participants felt that the modified wrench was the least physically intense technique, while the regular wrench restricted was ranked as third in least physical intensity. Furthermore, the modified wrench was the most efficient in opening the valve, and the regular wrench restricted was the least efficient. So in cases of emergency conditions where numerous valves are to be opened or closed quickly, the modified wrench would be the optimal technique to use for torques close to $25 \mathrm{Nm}$, in that it is the most efficient and comfortable technique. Also, the EMG results support it to be one of the least physically demanding techniques at the low torque.

In the case of the $50 \mathrm{Nm}$ torque, it was difficult to determine from the EMG results which method required the least overall muscle activity. However, one fact that was quite apparent from the EMG results was that the modified wrench seemed to require the most overall muscle activity in opening the valve because it had close to the highest \%MVC for most of the muscles. Although the modified wrench was the fastest technique to use at the $25 \mathrm{Nm}$ torque, the results indicate that it should be avoided at higher torques.

One limitation of this study is that the participants were not actual plant operators, but rather college students. Since the students were not experienced with valve wheel operations, they may not have been as efficient in turning the handwheel as an experienced operator or other workers who are accustomed to turning valve handwheels. In order to obtain consistent results, a protocol was established for each method of turning the wheel. With time and experience, some subjects may have preferred to adopt a slightly different posture, stance, or technique that could be faster, more efficient, or more comfortable than the method used in the trials. 


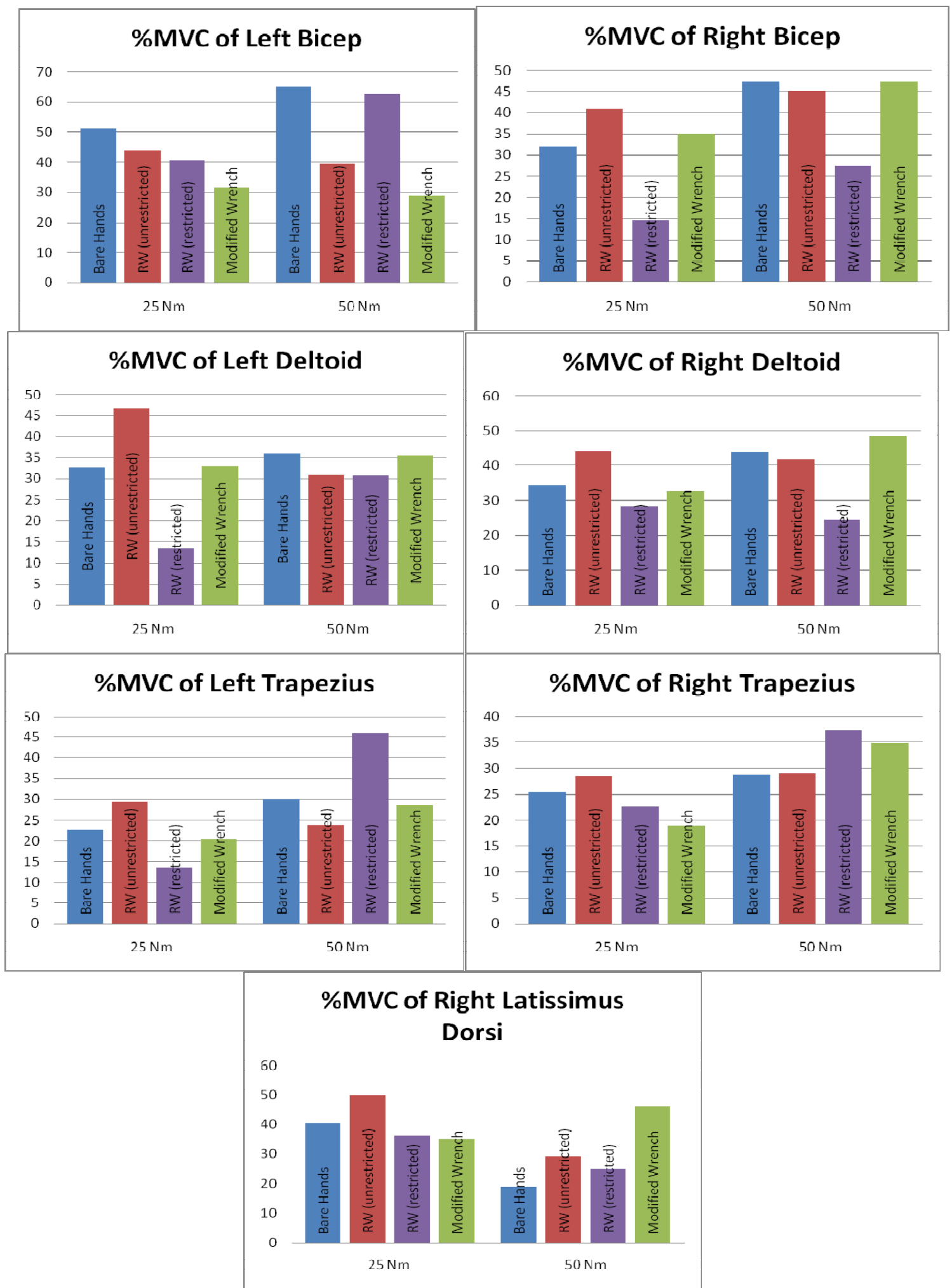

Figure 5. EMG results of \% MVC for each muscle 
Another limitation is that the sheer number of trunk, shoulder, and arm muscle co-contractions involved in turning a handwheel makes it difficult to interpret the EMG results with complete accuracy. Hof and Van Den Berg [2] suggest EMG is linearly related to the muscle force, but non-linear correlations may be due to the influence of muscle cocontraction on a joint. With the changes in posture, position, and muscle involvement while turning a valve wheel, measuring all of the muscle activity would be difficult. This would be due to the sheer number of muscles involved and input of forces from muscles below the ones being measured.

During experimentation, participants commented that the modified wrench was a bit uncomfortable

\section{References}

[1] Amell, T.K. and Kumar, S., 2001. Industrial handwheel actuation and the human operator: a review. International Journal of Industrial Ergonomics 28 5, pp 291-302.

[2] Hof A.L. and Van Den Berg, J.W. (1977) Linearity between the weighted sum of the human triceps surae and the total torque. Journal of Biomechanics 10, 529-539.

[3] Hoff, E.B, 2000. Ergonomic Evaluation of Manually Operated Valves. Dissertation, Louisiana State University, Department of Industrial Engineering.

[4] Jackson, A.S., Osburn, H.G., Laughery, K.R., Vaubel, K.P., 1992. Validity of isometric strength tests for predicting the capacity to crack, open and close industrial valves. Proceedings of the $\mathrm{Hu}-$ man Factors Society 36th Annual Meeting. pp. 688-691.

[5] Kumar, S., Mital, A. Electromyography in Ergonomics. 1996. Taylor \& Francis Ltd., London, England.

[6] Meyer, J.P., Lodde, B., Didry, G., and Horwat, F., 1999. Cardiorespiratory and subjective strains during actuation of large handwheels. due to the friction between the hand and the handle while rotating the handwheel. It is likely that a rotating handle would alleviate the discomfort and would give the modified wrench a better subjective rating at higher torques. A future study can determine how the rotating handle on the modified wrench could improve comfort.

One advantage of the modified wrench is that it could be used as the regular wrench restricted or unrestricted. Since the handle has a hinge in it, it could be straightened out like the regular wrench and be used like the regular wrench.

International Journal of Industrial Ergonomics 26 1, pp.47-56.

[7] Parks, S.C. and Schulze, L.J.H., 1998. The effects of valve wheel size, operation position and in-line pressures on required torque for gate valves. Process Safety Progress 17 4, pp. 263271.

[8] Robertson, R.J., and B.J. Noble, 1997 Perception of physical exertion: methods, mediators, and applications. Exercise and Sport Sciences Reviews 25: 407-452.

[9] Schulze, L.J.H., Goldstein, D. Patel, A. Stanton, E. and Woods, J., 1997. Torque Production using handwheels of different size during a simulated valve operation task. International Journal of Occupational Safety and Ergonomics 3 3-4, pp. 109-118.

[10] Wood, K.K., Schulze, L.J.H., Chen, J.C., Cleveland, T.G., 1999/2000. The effects of handwheel position on torque production capability of operators. Occupational Ergonomics 2(1), 53-65.

[11] Yoonton, Sarakorn, 1999. The ergonomic analysis of valve adjustment tasks for refinery unit operators at Koch petroleum group, St. Paul, Minnesota. Unpublished Masters research paper. 